\title{
IBM-1 Model ile çift-çift Fe İzotoplarının Nükleer Özelliklerinin İncelenmesi
}

\author{
Gül ÇAKIR ORUÇ', Mahmut BÖYÜKATA² \\ ${ }^{1}$ Kırıkkale Üniversitesi, Fen Bilimleri Enstitüsü, Fizik Anabilim Dalı, TR-71450, Kırıkkale \\ ${ }^{2}$ Kırıkkale Üniversitesi, Fen-Edebiyat Fakültesi, Fizik Anabilim Dalı, TR-71450, Kırıkkale \\ (ORCID: 0000-0001-5355-0899) (ORCID: 0000-0002-8065-9993)
}

\begin{abstract}
Öz
Bu çalışmada, A 60 bölgesinde bulunan bazı çift-çift Fe izotoplarının nükleer yapı özellikleri etkileşen bozon modeli-1 (IBM-1) ile incelenmiştir. Bu incelemede, çift-çift Fe izotoplarının enerji seviyeleri ve elektromanyetik geçiş oranları hesaplanmıştır. Hesaplamalar, temel banttaki ilk $4_{1}^{+}$ve $2_{1}^{+}$seviyelerinin oranlarını $\left(\mathrm{R}_{4^{+} / 2^{+}}\right)$, deneysel verileri bilinen diğer enerji seviyelerini, B(E2) geçiş değerlerini ve bunların oranlarını içermektedir. Öncelikle, incelenen izotopların deneysel enerji oranlarına bakılarak uygun model Hamiltonyeni oluşturulmuş ve sonra bu Hamiltonyenin parametreleri deneysel verilerden yola çıkılarak türetilmiştir. Daha sonra, bu izotopların enerji seviyeleri ve $B(E 2)$ geçiş değerleri hesaplanmış ve deneysel veriler ile karşılaştırılmıştır. Ayrıca, yapısal davranışları hakkında fikir sahibi olmak için ${ }^{50,52,56,58,60,62,64,66,68,70} \mathrm{Fe}$ izotoplarının $\mathrm{R}_{4^{+} / 2^{+}}=E\left(4_{1}^{+}\right) / E\left(2_{1}^{+}\right), \mathrm{R}_{0^{+} / 2^{+}}=$ $E\left(0_{2}^{+}\right) / E\left(2_{1}^{+}\right), \mathrm{R}_{1}(\mathrm{E} 2)=B\left(E 2: 4_{1}^{+} \rightarrow 2_{1}^{+}\right) / B\left(E 2: 2_{1}^{+} \rightarrow 0_{1}^{+}\right)$ve $\mathrm{R}_{2}(\mathrm{E} 2)=B\left(E 2: 0_{2}^{+} \rightarrow 2_{1}^{+}\right) / B\left(E 2: 2_{1}^{+} \rightarrow 0_{1}^{+}\right)$ oranlarının nötron sayılarına göre değişimi incelenmiştir.
\end{abstract}

Anahtar kelimeler: Enerji seviyeleri, Elektromanyetik geçişler, Etkileşen bozon modeli-1 (IBM-1), Fe izotopları.

\section{Investigation of Nuclear Properties of even-even Fe Isotopes within the IBM-1 Model}

\begin{abstract}
In this study, the nuclear structure properties of some even-even Fe isotopes in the A 60 region were investigated within the interacting boson model-1 (IBM-1). The energy levels and electromagnetic transmission rates of eveneven $\mathrm{Fe}$ isotopes were calculated in this investigation. The calculations include the ratios of the first $4_{1}^{+}$and $2_{1}^{+}$ energy levels $\left(\mathrm{R}_{4^{+} / 2^{+}}\right)$, the other experimentally known energy levels, the $\mathrm{B}(\mathrm{E} 2)$ values and their ratios. First of all, a suitable model Hamiltonian was constructed and later the parameters of this Hamiltonian derived from the experimental data. Afterwards, the energy levels and $\mathrm{B}(\mathrm{E} 2)$ values of these isotopes were calculated and then compared with experimental data. Furthermore, changing of $\mathrm{R}_{4^{+} / 2^{+}}=E\left(4_{1}^{+}\right) / E\left(2_{1}^{+}\right), \mathrm{R}_{0^{+} / 2^{+}}=E\left(0_{2}^{+}\right) / E\left(2_{1}^{+}\right)$, $\mathrm{R}_{1}(\mathrm{E} 2)=B\left(E 2: 4_{1}^{+} \rightarrow 2_{1}^{+}\right) / B\left(E 2: 2_{1}^{+} \rightarrow 0_{1}^{+}\right) \quad$ and $\mathrm{R}_{2}(\mathrm{E} 2)=B\left(E 2: 0_{2}^{+} \rightarrow 2_{1}^{+}\right) / B\left(E 2: 2_{1}^{+} \rightarrow 0_{1}^{+}\right)$ratios of $50,52,56,58,60,62,64,66,68,70 \mathrm{Fe}$ isotopes were investigated according to the neutron numbers to have an idea their structural behavior.
\end{abstract}

Keywords: Electromagnetic transitions, Energy levels, Interacting Boson Model, Fe isotopes.

\section{Giriş}

Kararlılık eğrisi boyunca, atom çekirdekleri birbirinden farklı davranışlar ve özellikler sergilemektedir. Deneysel sonuçlar [1] incelendiğinde, bu farkl1lıklar kararlılık eğrisinde bölgelere göre ve izotop, izoton, izobar çizgileri boyunca gözlenebilmektedir. Son yıllarda, A 50 bölgesinde yer alan orta kütleli çift-çift Fe izotopları için ile yapılan deneysel çalışmaların yanında, kabuk modeli, QRPN, etkileşen bozon modeli (IBM) gibi bazı modeller ile çalışmalar gerçekleştirilmiştir [2-9]. Çift-çift ${ }^{50,52,54,56,58,60} \mathrm{Fe}$ izotoplarının kuantum şekil faz geçişleri teorik olarak incelenmiştir [2]. ${ }^{52} \mathrm{Fe}$ ve ${ }^{62,64,66} \mathrm{Fe}$ izotoplarının $2^{+}$

*Sorumlu yazar: gulusckr19@gmail.com

Geliş Tarihi: 28.12.2020, Kabul Tarihi: 01.03.2021 
seviyeleri ile B(E2) geçişleri kabuk model ile hesaplanmıştır [3] ve ilerleyen y1llarda ${ }^{50,52} \mathrm{Fe}$ ve ${ }^{62-68} \mathrm{Fe}$ izotopları için bu hesaplamalar tekrarlanmıştır [4]. Fe çekirdeğinin, $\mathrm{N}=28-50$ sihirli sayıları arasında yer alan bütün çift-çift izotoplarının $2^{+}$enerjileri ve $\mathrm{B}\left(\mathrm{E} 2 ; 2^{+} \rightarrow 0^{+}\right)$değerleri kabuk modeli ile incelenmiştir [5,6]. Bu Fe izotoplarının ve komşu Cr izotoplarının kollektif özellikleri etkileşen kabuk model ve etkileşen bozon modeli-2 (IBM-2) ile incelenmiştir [7]. Ortalama alan yaklaşım modelleri ile ${ }^{56-68} \mathrm{Fe}$ izotoplarının uyarılmış enerji durumları, E2 geçişlerinin yanında deformasyonları da incelenmiştir [8]. Kabuk modelinin Hilbert uzayı çerçevesinde ${ }^{52} \mathrm{Fe}$ izotopunun uyarılmış enerji seviyeleri ve enerji oranları Hamiltonyen parametreleri değiştirilerek nükleer şekil geçişleri incelenmiştir [9]. Son y1llarda Fe izotopları üzerine yapılan teorik çalışmalara bakıldığında, ağırlıklı olarak kabuk model ile yapıldığı görülmektedir. Bu çalışmada, A 60 bölgesinde bulunan demir çekirdeğinin bilenen bütün çift-çift $\mathrm{Fe}$ izotopları etkileşen bozon modeli-1 (IBM-1) ile incelenmiştir. IBM-1 ile yapılan bu çalışma, temel banttaki uyarılmış $4_{1}^{+}$ve $2_{1}^{+}$seviyelerinin oranları yani $E\left(4_{1}^{+}\right) / E\left(2_{1}^{+}\right)$, diğer uyarılmış enerji seviyelerinin ve $\mathrm{B}(\mathrm{E} 2)$ geçiş değerlerinin hesaplamalarını içermektedir. Ayrıca, $E\left(0_{2}^{+}\right) / E\left(2_{1}^{+}\right)$, $B\left(E 2: 4_{1}^{+} \rightarrow 2_{1}^{+}\right) / B\left(E 2: 2_{1}^{+} \rightarrow 0_{1}^{+}\right)$ve $B\left(E 2: 0_{2}^{+} \rightarrow 2_{1}^{+}\right) / B\left(E 2: 2_{1}^{+} \rightarrow 0_{1}^{+}\right)$oranlarının nötron sayısına göre şekilsel değişimi analiz edilmiştir.

Makale içeriği şu şekilde düzenlenmiştir; giriş bölümünde, incelenen çekirdekler üzerine son yıllarda yapılan çalışmalar hakkında kısaca bilgi verilmiştir ve bu çalışmanın düzeni özetlenmiştir. İkinci bölümde, materyal ve yöntem açıklanmıştır, burada kullanılan Hamiltonyen ve diğer formülasyonlar alt başliklar halinde sunulmuştur. Üçüncü bölümde, elde edilen sonuçlar tablo ve grafikler ile verilmiştir. A 60 bölgesindeki incelenen çift-çift Fe izotopları için oluşturulan Hamiltonyen ve elde edilen parametreler verilmiştir. Hesaplanan uyarılmış enerji seviyeleri ve $B(E 2)$ değerleri grafikler ile verilmiş ve deneysel veriler ile karşılaştırılmıştır. Ayrıca enerji oranları ve E2 geçiş oranlarının nötron sayısına göre değişimi şekiller ile verilmiştir. Bu izotopların yapısal davranışları dinamik simetrileri ve kritik noktaları dikkate alınarak tartışılmıştır. Son bölümde, sonuçların yorumları özetlenmiştir. Elde edilen hesaplamalar ile deneysel sonuçların uyumlu olduğu görülmüsstür.

\section{Materyal ve Metot}

Nükleer çekirdeklerin sergiledikleri yapısal özellikleri açıklamak için çeşitli yaklaşımlar ortaya konmuştur [10]. Bu yaklaşımlardan bir tanesi de Lie cebri üzerine kurulmuş olan yani grup teoriksel bir yaklaşım olan etkileşen bozon model (IBM)'dir [11]. U(6) grubu üzerine kurulmuş olan bu model, orta ve ağır kütleli çift-çift çekirdeklerde oldukça başarılı sonuçlar vermektedir. Günümüzde, çekirdeklerin yapısal özelliklerini açıklamak için aktif olarak farklı modeller ile birlikte kullanılmakta ve gelişimini devam ettirmektedir [12-15]. Günümüzde hala kullanılan ve sihirli sayıya sahip olmayan çift proton-çift nötron sayısına sahip çekirdeklerin açıklanmasında oldukça başarılıdır. Bu cebirsel modelde, incelenen çekirdek için sihirli sayılar arasındaki bozonların etkileşmesi dikkate alınır. Bozon sayısı yakın kapalı kabuklar dikkate alınarak hesaplanmaktadır.

\subsection{Etkileşen Bozon Modeli-1 (IBM-1)}

Etkileşen bozon yaklaşımı olarak adlandırılan IBM modeli, ilk olarak Feshbach ve Iachello tarafından ortaya konmuş ve çift-çift ${ }^{16} \mathrm{O}$ çekirdeğine uygulanmıştır $[16,17]$. İlerleyen yıllarda, Arima ve Iachello tarafından geliştirilirmiştir [12]. Çift-çift çekirdeklerin düşük kolektif durumlarının açıklanması için kurulan bu yaklaşımda, proton ve nötronlar nükleon olarak ele alınır ve IBM-1 model olarak adlandırılır. En temel versiyon olan IBM-1 modeli, $L=0$ ve $L=2$ açısal momentumları dikkate alınır ve bu açısal momentumlara karşılık gelen $s$ ve $d$ bozonlarının etkileşimi üzerine kurulmuştur. sd-IBM olarak da adlandırılan bu modelde, $L=0(\mu=0)$ 'dan kaynaklanan bir tane monopole $S^{\dagger}$ bozon işlemcisi ve $L=$ $2(\mu=-2,-1,0,+1,+2)$ 'den kaynaklanan beş tane kuadrupole $d_{\mu}^{\dagger}$ bozon işlemcisi olmak üzere toplam altı işlemci mevcuttur. Bu altı işlemci, U(6) grubuna karşılık gelmektedir ve bundan dolayı IBM modeli $\mathrm{U}(6)$ grubu üzerine kurulmuştur. Bu grubun mümkün olan üç tane $\mathrm{U}(5), \mathrm{SU}(3), \mathrm{O}(6)$ alt grubu vardır ve bunlar dinamik simetriler olarak adlandırılır. İncelenen çekirdeğin geometrisiyle de alakalı olan bu U(5)-titreşim, SU(3)-rotasyon (dönme) ve O(6)- $\gamma$-kararsız limitleri sırasıyla küresel, eksenel deforme (prolate/oblate) ve $\gamma$-kararsız çekirdeklere karşılık gelmektedir. Küreselden deforme geçiş bölgesi arasında bulunan çekirdekleri model çerçevesinde incelemek mümkündür. IBM'in karakteristik 
özellikleri grup teori ile üretildiği için bahsedilen $\mathrm{U}(5), \mathrm{SU}(3), \mathrm{O}(6)$ limitler için analitik çözümler ayrı ayr1 ortaya konmuştur [11,12]. Ayrica bu limitler arasında meydana gelen E(5) ve X(5) kritik nokta simetrileri Iachello tarafindan ortaya konmuştur $[18,19]$.

En yaygin kullanılan IBM-1 Hamiltonyeninin genel formu [12]

$$
H=\varepsilon^{\prime \prime} \hat{n}_{d}+a_{0} \hat{P}^{\dagger} \hat{P}+a_{1} \widehat{L}^{2}+a_{2} \widehat{Q}^{2}+a_{3} \widehat{T}_{3}^{2}+a_{4} \widehat{T}_{4}^{2}
$$

ile verilmektedir. Burada, $\hat{n}_{d}$ bozon işlemcisi olup diğer $\hat{P}, \hat{L}, \hat{Q}, \widehat{T}_{3}$ ve $\widehat{T}_{4}$ terimleri sırasiyla çiftlenim, açısal momentum, kuadropol, oktupol ve hegzadepol işlemcileridir. Hamiltonyende ki katsayılar ise serbest parametreleri ifade etmektedir.

IBM-1'de $E 2$ elektromanyetik geçiş operatörünün genel formu [11],

$$
\hat{T}^{(E 2)}=\alpha_{2}\left[\hat{d}^{\dagger} \times \tilde{s}+\hat{s}^{\dagger} \times \tilde{d}\right]^{(2)}+\beta_{2}\left[\hat{d}^{\dagger} \times \tilde{d}\right]^{(2)}
$$

şeklindedir. Burada, $\hat{d}$ ve $\hat{s}$ bozon işlemcileridir, $\alpha_{2}$ ve $\beta_{2}$ ise serbest parametrelerdir. Uyarılmış seviyeler arasındaki elektriksel geçiş olasılıklarının yani B(E2)'nin değerleri

$$
B\left(E 2 ; L_{i} \rightarrow L_{s}\right)=\frac{1}{2 L_{i}+1}\left|\left(\left\langle L_{s}\left\|\hat{T}^{(E 2)}\right\| L_{i}\right\rangle\right)\right|^{2}(e b)^{2}
$$

ile azaltılmış matris elemanlarına bağlı olarak verilmektedir.

\section{Bulgular ve Tartışma}

IBM-1 modeli ile incelenen ${ }^{50,52,56,58,60,62,64,66,68,70} \mathrm{Fe}$ izotoplar1 karalılık eğrisinin A $\sim 50$ bölgesinde bulunmaktadır. ${ }^{54} \mathrm{Fe}$ izotopunun nötron sayısı 28 'dir ve bu sihirli sayı olduğu için nötron bozon sayısı sıfır. Bundan dolayı toplan bozon sayısı hesaplanamayacağı için hesaplara dâhil edilmemiştir. $50,52,56,58,60,62,64,66,68,70 \mathrm{Fe}$ izotoplarının nötron sayıları sırasıyla 24, 26, 30, 32, 34, 36, 38, 40, 42, 44'dür. Model çerçevesinde incelenen çekirdeğin bozon sayıları en yakın sihirli sayı dikkate alınarak hesaplanır. Her bir nükleon çifti bir bozona karşılık gelir ve bu şekilde bütün Fe izotoplarının hesaplanan bozon sayıları Tablo 1'de sunulmuştur. İncelenen çekirdeğin nükleer yapısal davranışlarını anlamak için öncelikle, temel bantta yer alan ilk iki uyarılmış $4_{1}^{+}$ve $2_{1}^{+}$seviyelerinin enerji oranlarına $\left(\mathrm{R}_{4^{+} / 2^{+}}\right)$ bakılabilir. $\mathrm{Bu}$ izotopların deneysel $\mathrm{R}_{4^{+} / 2^{+}}$oranlarının nötron sayılarına göre değişimi Şekil 1 'de verilmiştir.

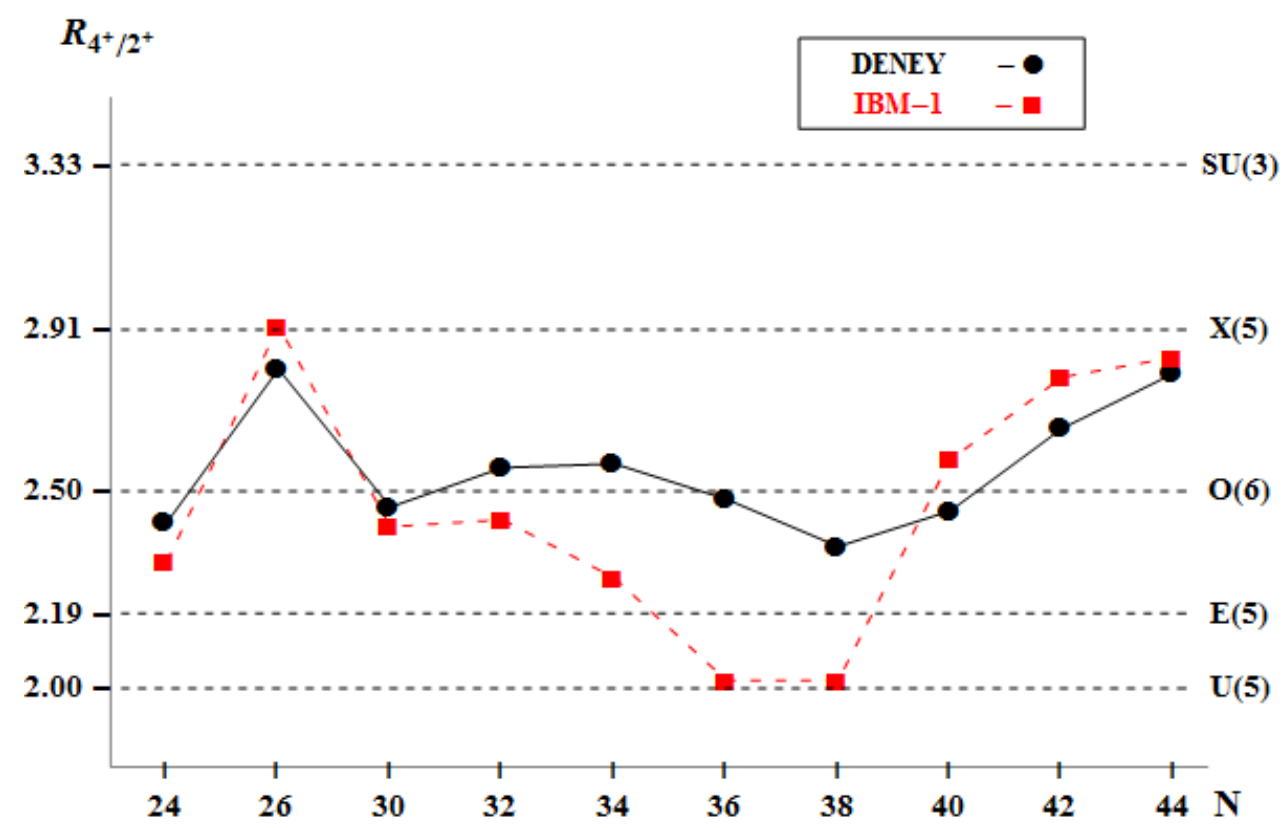

Şekil 1. Çift-çift Fe izotoplarının deneysel [1] ve hesaplanan $\mathrm{R}_{4^{+} / 2^{+}}$oranlarının nötron sayılarına göre değişimi 
Görüldüğg̈ gibi, Şekil 1, modelin dinamik simetrilerinin yani U(5), SU(3), O(6) limitlerinin ve X(5), E(5) kritik noktalarının tipik enerji oranları değerlerini de içermektedir. Şekil incelendiğinde, ${ }_{50,56,58,60,62,66} \mathrm{Fe}$ izotoplarının deneysel enerji oranları $\mathrm{O}(6)$ limitine yakın olup $\gamma$-kararsız şekil sergileyebileceği öngörülebilir. ${ }^{52,68,70} \mathrm{Fe}$ izotoplarının enerji oranı $\mathrm{X}(5)$ kritik noktasına yakın olup geçiş bölgesindedir. ${ }^{64} \mathrm{Fe}$ izotopu ise $\mathrm{O}(6)$ ile $\mathrm{E}(5)$ kritik noktası arasında yer almaktadır.

$\mathrm{Bu}$ izotopların, uyarılmış enerji seviyelerini hesaplamak için IBM-1 modeli çerçevesinde yazılan PHINT kodu kullanılmıştır. Hesaplamalarda kullanılan model Hamiltonyeni ise aşağıdaki şekildedir;

$$
H=(\text { eps }) \hat{n}_{d}+(\text { pair }) \hat{P}^{\dagger} \hat{P}+(e l l) \hat{J}^{2}+(q q) \hat{Q}^{2}+(\text { oct }) \widehat{T}_{3}^{2}+(\text { hex }) \widehat{T}_{4}^{2}
$$

burada verilen katsayılar denklem (1)'de verilen Hamiltonyenin katsayıları olup serbest parametreleri temsil etmektedir ve PHINT kodunun [20] giriş parametreleri formatında yazılmıştır. Bu çalışma çerçevesinde, incelenen ${ }^{50,52,56,58,60,62,64,66,68,70} \mathrm{Fe}$ izotoplar1 için üretilen serbest Hamiltonyen parametreleri Tablo 1'de verilmiştir.

Tablo 1. ${ }^{50,52,56,58} \mathrm{Fe}$ izotoplarının bozon sayıları $\left(\mathrm{N}^{*}\right)$ ve IBM-1'de kullanılan Hamiltonyen parametreleri

\begin{tabular}{ccccccccccc}
\hline PHINT & ${ }^{50} \mathrm{Fe}$ & ${ }^{52} \mathrm{Fe}$ & ${ }^{56} \mathrm{Fe}$ & ${ }^{58} \mathrm{Fe}$ & ${ }^{60} \mathrm{Fe}$ & ${ }^{62} \mathrm{Fe}$ & ${ }^{64} \mathrm{Fe}$ & ${ }^{66} \mathrm{Fe}$ & ${ }^{68} \mathrm{Fe}$ & ${ }^{70} \mathrm{Fe}$ \\
\hline $\mathrm{N}$ & 3 & 2 & 2 & 3 & 4 & 5 & 6 & 6 & 5 & 4 \\
eps & 0.1 & 0.26 & 0.72 & - & 0.03 & 0.828 & 0.94 & 0.32 & 0.21 & 0.179 \\
pair & - & - & - & 0.285 & 0.9 & - & - & - & 0.001 & 0.001 \\
oct & 0.08 & - & - & 0.091 & 0.115 & 0.0285 & - & - & - & - \\
qq & - & - & -0.4 & - & - & - & -0.01 & - & - & - \\
ell & 0.0425 & 0.19 & -0.085 & 0.0672 & 0.035 & -0.001 & - & 0.08 & 0.1 & 0.1 \\
chq & - & - & -2.1 & - & - & - & -3.07 & - & - & - \\
\hline
\end{tabular}

$* \mathrm{~N}$ bozon sayısıdır (nötron sayısı değil)

Tablo 1 incelendiğinde, ${ }^{50,58,62,64,68,70} \mathrm{Fe}$ izotopları için üç parametre, ${ }^{52,66} \mathrm{Fe}$ izotopları için iki parametre ve ${ }^{56,60} \mathrm{Fe}$ izotopları için dört parametre kullanılmıştır. Bu parametreler ile hesaplanan sonuçlar Şekil 2'de verilmiştir.

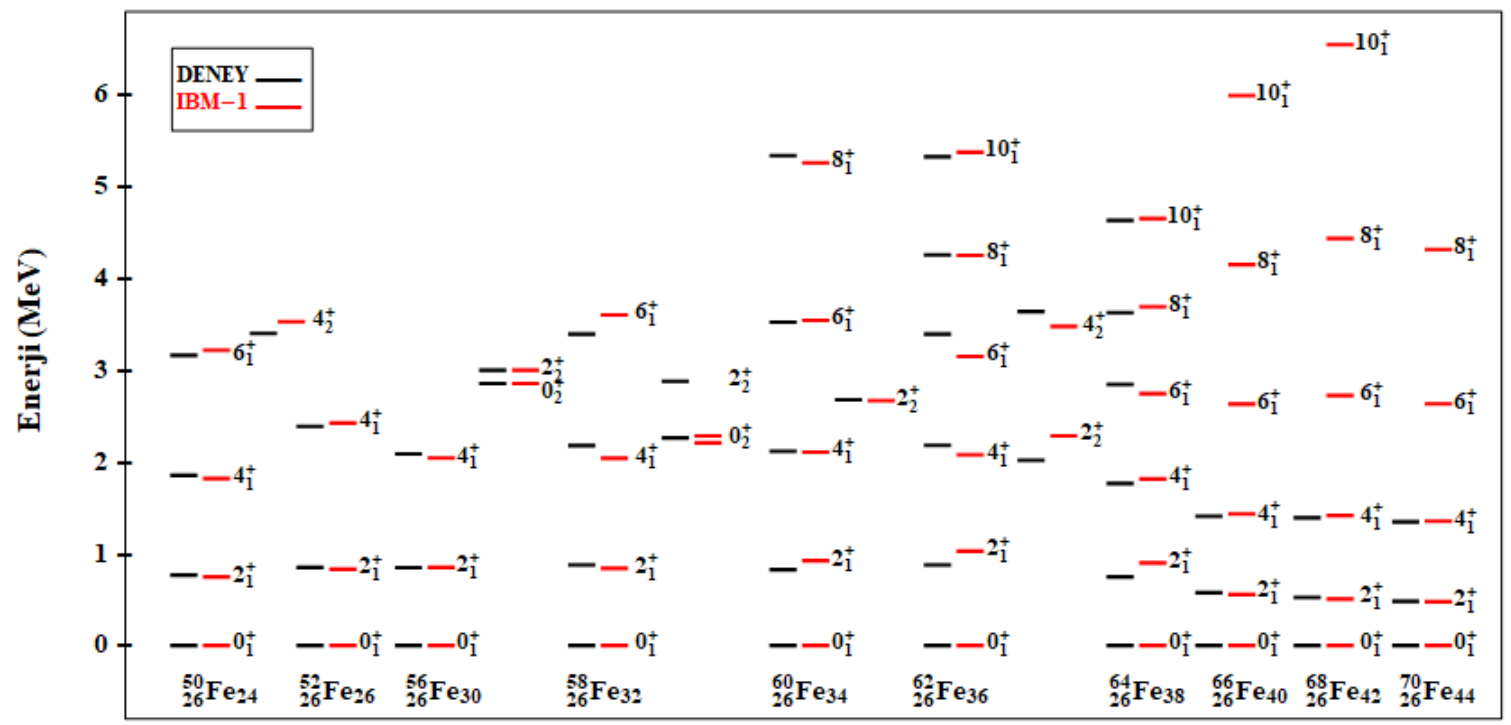

Şekil 2. ${ }^{50,52,56,58,60,62,64,66,68,70} \mathrm{Fe}$ izotoplarının deneysel [1] ve hesaplanan uyarılmış enerji spektrumları

Şekil 2 incelenen ${ }^{50,52,56,58,60,62,64,66,68,70} \mathrm{Fe}$ izotoplarının hesaplanan ve deneysel [1] uyarılmış enerji seviyelerini içermektedir. Şekil incelendiğinde, hesaplanan sonuçların deneysel veriler ile genelde uyumlu olduğu görülmektedir. IBM-1 modeli ile bozon sayının iki katına kadar olan seviyeler hesaplanabilmektedir. Örneğin, ${ }^{50} \mathrm{Fe}$ izotopunun bozon sayıs1 $\mathrm{N}=3$ olduğundan, temel bantta $6_{1}^{+}$ 
seviyesine kadar olan seviyeler hesaplanabilmiştir. Aynı nedenden dolayı, ${ }^{58} \mathrm{Fe}$ izotopunun da $6_{1}^{+}$ seviyesine kadar olan uyarılmış durumların hesabı yapılmıştır. Diğer ${ }^{52} \mathrm{Fe}$ ve ${ }^{56} \mathrm{Fe}$ izotoplarının bozon sayısı iki olduğu için uyarılmış $4_{1}^{+}$seviyesine kadar hesap gerçekleştirilmiştir. Diğer izotoplarında enerji seviyeleri bozon sayılarına göre hesaplanmıştır. ${ }^{50,56,58,60,62} \mathrm{Fe}$ izotoplarının diğer bantlarda yer alan ve deneysel olarak bilinen uyarılmış seviyeleri de hesaba dâhil edilmiştir. ${ }^{52} \mathrm{Fe}$ izotopunun, sadece $2_{1}^{+}$ve $4_{1}^{+}$uyarılmış seviyelerinin hesabı gerçekleştiği için Tablo 1'de görüldüğü üzere az sayıda parametre kullanılmıştır. Diğer izotoplarda ise temel bant dışında ki uyarılmış seviyeler de hesaba dâhil edilmiştir ve bundan dolayı daha fazla parametreye ihtiyaç duyulmuştur.

Hesaplanan $\mathrm{R}_{4^{+} / 2^{+}}$oranları Şekil 1'e de eklenmiştir, görüldüğü üzere, bunların da deneysel veriler ile uyumlu olduğu görülmekte ve birbirini desteklemektedir. Dolayısıyla bu uyum, Hamiltonyen parametrelerinin uygun olduğunu göstermektedir. Şekil 1'de verilen, hesaplanan enerji oranları incelendiğinde ${ }^{56,58,66} \mathrm{Fe}$ izotoplarının $\mathrm{O}(6)$ limitine yakınken ${ }^{62,64} \mathrm{Fe}$ izotoplarının $\mathrm{U}(5)$ limitine yakın olduğu görülmektedir. ${ }^{50,60} \mathrm{Fe}$ izotopları $\mathrm{O}(6)$ limiti ile $\mathrm{E}(5)$ kritik noktası arasında yer alırken, ${ }^{52,68,70} \mathrm{Fe}$ izotopları ise $\mathrm{X}(5)$ kritik noktasına yakınındadır, dolayısıyla IBM-1 ile hesaplanan enerji oranlarına göre bu izotopların geçiş bölgesinde yer aldığına işaret etmektedir. Sadece $\mathrm{R}_{4^{+} / 2^{+}}$oranına bakmak, incelenen Fe izotoplarının yapısal davranışını anlamak için yeterli değildir, bunun dışında, $\mathrm{R}_{0^{+} / 2^{+}}$ oranlarının nötron sayısına göre değişimi Şekil 3'de sunulmuştur.

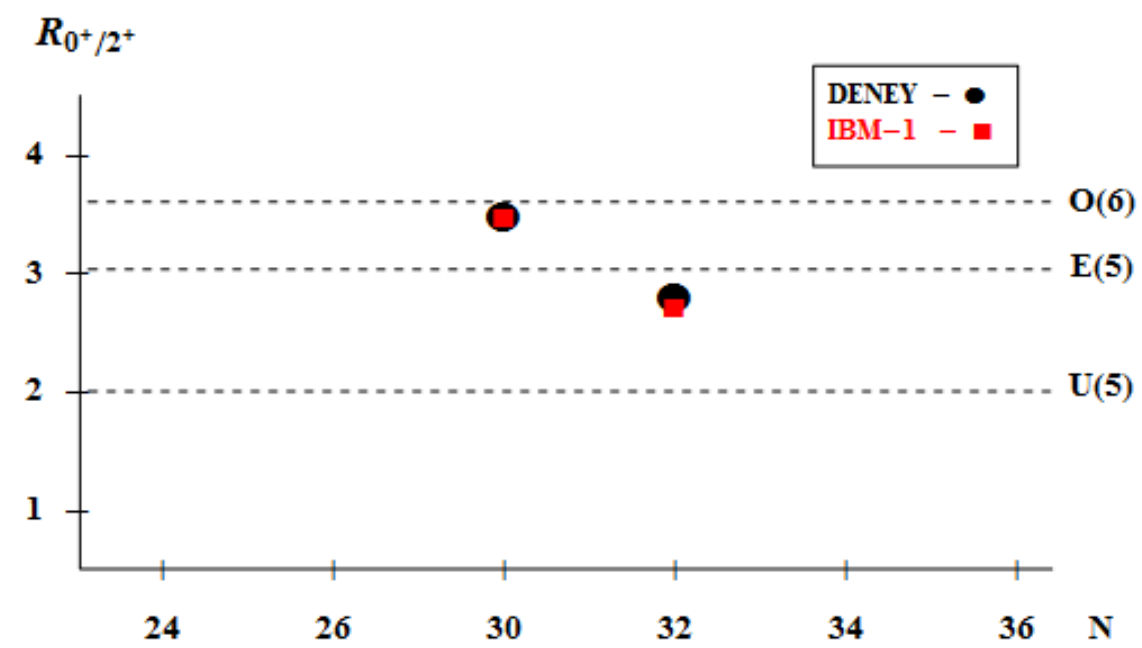

Şekil 3. ${ }^{56,58} \mathrm{Fe}$ izotoplarının deneysel [1] ve hesaplanan $\mathrm{R}_{0^{+} / 2^{+}}$oranlarının nötron sayılarına göre değişimi.

Şekil 3, U(5)-O(6) limitleri ve $\mathrm{E}(5)$ kritik noktasının $\mathrm{R}_{0^{+} / 2^{+}}$oranlarının tipik değerleri ve deneysel sonuçları bilinen ${ }^{56,58} \mathrm{Fe}$ izotoplarının enerji oranlarını içermektedir. Şekilden de anlaşılacağ üzere bu iki izotop E(5) kritik noktasına yakındır. Hesaplanan sonuçlarda deneysel verileri doğrulamaktadır. Buna göre bu izotoplar, $\mathrm{U}(5)-\mathrm{O}(6)$ arasında yer alan $\mathrm{E}(5)$ kritik noktası civarındadır.

Uyarılmış enerji seviyeleri kadar elektromanyetik geçiş oranları da nükleer yapıyı anlamak için önemlidir. IBM-1 model çerçevesinde, B(E2) geçişlerinin hesaplanmasında, (2) numaralı denklemde verilen serbest parametrelerin elde edilmesi için incelenen izotopların her biri için deneysel $B\left(E 2: 2_{1}^{+} \rightarrow 0_{1}^{+}\right)$geçiş değerleri referans alınmıştır. (2) numaralı eşitlikte verilen $E 2$ elektromanyetik geçiş operatörü,

$$
\widehat{T}^{(E 2)}=E 2 S D\left[\hat{d}^{\dagger} \times \tilde{s}+\hat{s}^{\dagger} \times \tilde{d}\right]^{(2)}+E 2 D D\left[\hat{d}^{\dagger} \times \tilde{d}\right]^{(2)}
$$

şeklinde tekrar yazılabilir. Buradaki katsayılar, PHINT-PBEM kodunda yer alan serbest parametrelere karş1lık gelmektedir. Her izotop için üretilen parametreler Tablo 2'de sunulmuştur.

Tablo 2. ${ }^{50,52,56,58} \mathrm{Fe}$ izotoplarının B(E2) değerleri için kullanılan parametreler

\begin{tabular}{lllllll}
\hline & \multicolumn{1}{c}{${ }^{50} \mathrm{Fe}$} & ${ }^{52} \mathrm{Fe}$ & \multicolumn{1}{c}{${ }^{56} \mathrm{Fe}$} & ${ }^{58} \mathrm{Fe}$ & ${ }^{60} \mathrm{Fe}$ & ${ }^{62} \mathrm{Fe}$ \\
\hline$E 2 S D$ & 0.098 & 0.0906 & 0.099 & 0.0768 & 0.0546 & 0.0642 \\
$E 2 D D$ & -0.0317 & -0.0317 & -0.0317 & -0.0317 & -0.0317 & -0.0317 \\
\hline
\end{tabular}


Tablo 2'deki parametreler her bir izotopun diğer B(E2) geçiş değerlerinin hesabı içinde kullanılmıştır. Elde edilen sonuçlar Tablo 3'de sunulmuştur.

Tablo 3. Deneysel [1] ve hesaplanan B(E2) değerleri $\left(\times 10^{-2} \mathrm{e}^{2} \mathrm{~b}^{2}\right)$.

\begin{tabular}{cccc}
\hline Çekirdek & $\mathrm{J}_{\mathrm{i}} \rightarrow \mathrm{J}_{\mathrm{s}}$ & Deney & Hesap \\
\hline \multirow{3}{*}{${ }^{50} \mathrm{Fe}$} & $2_{1}^{+} \rightarrow 0_{1}^{+}$ & $2,845(0,657)$ & 2,88 \\
& $4_{1}^{+} \rightarrow 2_{1}^{+}$ & & 3,84 \\
& $6_{1}^{+} \rightarrow 4_{1}^{+}$ & & 2,88 \\
\hline${ }^{52} \mathrm{Fe}$ & $2_{1}^{+} \rightarrow 0_{1}^{+}$ & $1,637(0,219)$ & 1,64 \\
& $4_{1}^{+} \rightarrow 2_{1}^{+}$ & $2,998(0,692)$ & 1,64 \\
\hline${ }^{56} \mathrm{Fe}$ & $2_{1}^{+} \rightarrow 0_{1}^{+}$ & $2,138(0,089)$ & 2,14 \\
& $4_{1}^{+} \rightarrow 2_{1}^{+}$ & $3,054(0,636)$ & 1,77 \\
\hline \multirow{3}{*}{${ }^{58} \mathrm{Fe}$} & $2_{1}^{+} \rightarrow 0_{1}^{+}$ & $2,467(0,080)$ & 2,48 \\
& $4_{1}^{+} \rightarrow 2_{1}^{+}$ & $6,268(0,934)$ & 2,70 \\
& $6_{1}^{+} \rightarrow 4_{1}^{+}$ & $3,467(1,333)$ & 1,77 \\
\hline \multirow{3}{*}{${ }^{60} \mathrm{Fe}$} & $2_{1}^{+} \rightarrow 0_{1}^{+}$ & $1.898(0,195)$ & 1,91 \\
& $4_{1}^{+} \rightarrow 2_{1}^{+}$ & $1,953(0.558)$ & 2,30 \\
& $6_{1}^{+} \rightarrow 4_{1}^{+}$ & & 1,99 \\
& $8_{1}^{+} \rightarrow 6_{1}^{+}$ & & 1.19 \\
\hline & $2_{1}^{+} \rightarrow 0_{1}^{+}$ & $2,055(0,233)$ & 2,06 \\
& $4_{1}^{+} \rightarrow 2_{1}^{+}$ & & 3,30 \\
${ }^{62} \mathrm{Fe}$ & $6_{1}^{+} \rightarrow 4_{1}^{+}$ & & 3,71 \\
& $8_{1}^{+} \rightarrow 6_{1}^{+}$ & & 3,30 \\
& $10_{1}^{+} \rightarrow 8_{1}^{+}$ & & 2,06 \\
\hline
\end{tabular}

Tablo 3 incelendiğinde ve deneylerdeki hata paylarını da göz önünde tutulduğunda hesaplanan sonuçlar, özellikle $B\left(E 2: 2_{1}^{+} \rightarrow 0_{1}^{+}\right)$değerleri için, deneysel veriler ile oldukça uyumlu olduğu görülmektedir. Henüz deneysel olarak bilinmeyen B(E2) geçiş değerleri için tahmini olarak hesaplanan sonuçlarda tabloya eklenmiştir. B(E) geçişlerinin bazı oranlarının nötron sayısına göre değişimi Şekil 4 ve Şekil 5'de verilmiştir.

Şekil 4'de, temel bantta meydana gelen $\mathrm{B}\left(\mathrm{E} 2: 4_{1}^{+} \rightarrow 2_{1}^{+}\right)$geçişinin $\mathrm{B}\left(\mathrm{E} 2: 2_{1}^{+} \rightarrow 0_{1}^{+}\right)$geçişine oranının nötron sayısına göre değişim grafiği verilmiştir. Grafik incelendiğinde, deneysel verileri bilinen ${ }^{52,56,58,60} \mathrm{Fe}$ izotoplarının oranları ve bunların hata payları mevcuttur. Bu izotopların $R_{1}(E 2)$ oranları, IBM-1 model ile hesaplanmış olup, deneysel verisi bilinmeyen ${ }^{50,62} \mathrm{Fe}$ izotopları için de tahmin yapılmıştır. Ayrıca, Şekil 4 küresel şekilden deforme $\gamma$-kararsız şekle yani $U(5) \rightarrow O(6)$ 'ya geçişi ve bu iki şekil arasında bulunan aynı zamanda $\mathrm{E}(5)$ kritik noktanın $[18,21,22]$ ve ilgili dinamik simetrilerin tipik $R_{1}(E 2)$ oranı değerlerini içermektedir. Şekilden görüldüğ̈ gibi ${ }^{52} \mathrm{Fe}$ ve ${ }^{56} \mathrm{Fe}$ izotopları $\mathrm{E}(5)$ kritik noktasının yakınında yer almakta ve ${ }^{58,60} \mathrm{Fe}$ izotoplarının oranı bu bölgenin uzağındadır ancak ${ }^{60} \mathrm{Fe}$ izotopu $\mathrm{O}(6)$ limitine yakındır.

Şekil 5'de, $\mathrm{B}\left(\mathrm{E} 2: 0_{2}^{+} \rightarrow 2_{1}^{+}\right)$geçişinin $\mathrm{B}\left(\mathrm{E}: 2_{1}^{+} \rightarrow 0_{1}^{+}\right)$geçişine oranının nötron sayısına göre değişim grafiği verilmiştir. Deneysel veri olarak sadece ${ }^{56,58} \mathrm{Fe}$ izotoplarının değerleri vardır. Bu oranda da $\mathrm{E}(5)$ kritik noktasının tipik $\mathrm{R}_{2}$ (E2) oranı için farklı iki kaynaktan elde edilen değeri verilmiştir. Şekil 5 incelenen ${ }^{56,58} \mathrm{Fe}$ izotoplarının, farklı kaynaklardan [18,21,22] alınan üç farklı $\mathrm{E}(5)$ kritik nokta değerlerine göre davranışını göstermektedir. Grafik incelendiğinde, bu izotopların $\mathrm{R}_{2}(\mathrm{E} 2)$ oranları bakımından E(5) kritik noktası etrafında yer aldığını söylemek mümkündür. 


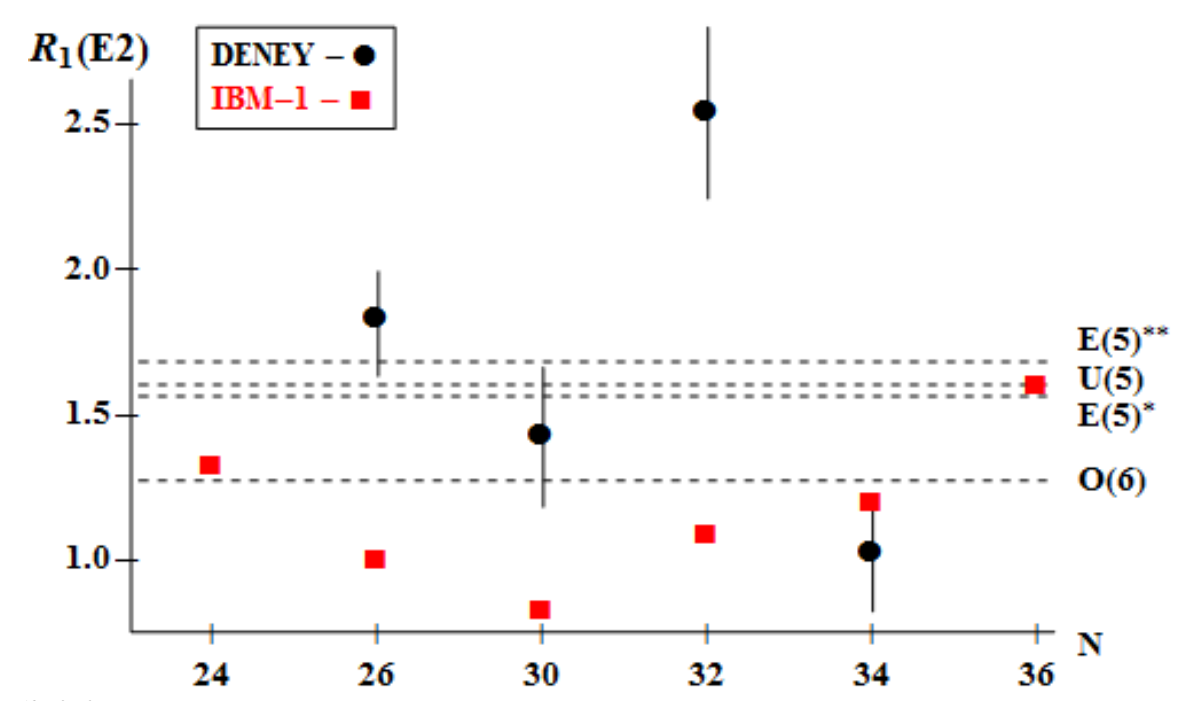

Şekil 4. ${ }^{50,52,56,58,60,62} \mathrm{Fe}$ izotoplarının $\boldsymbol{R}_{\mathbf{1}}(\boldsymbol{E} 2)=\boldsymbol{B}\left(\boldsymbol{E} 2: \mathbf{4}_{1}^{+} \rightarrow \mathbf{2}_{1}^{+}\right) / \boldsymbol{B}\left(\boldsymbol{E} 2: \mathbf{2}_{1}^{+} \rightarrow \mathbf{0}_{1}^{+}\right)$oranlarının nötron sayısına göre değişimi $(*[18,22], * *[21])$

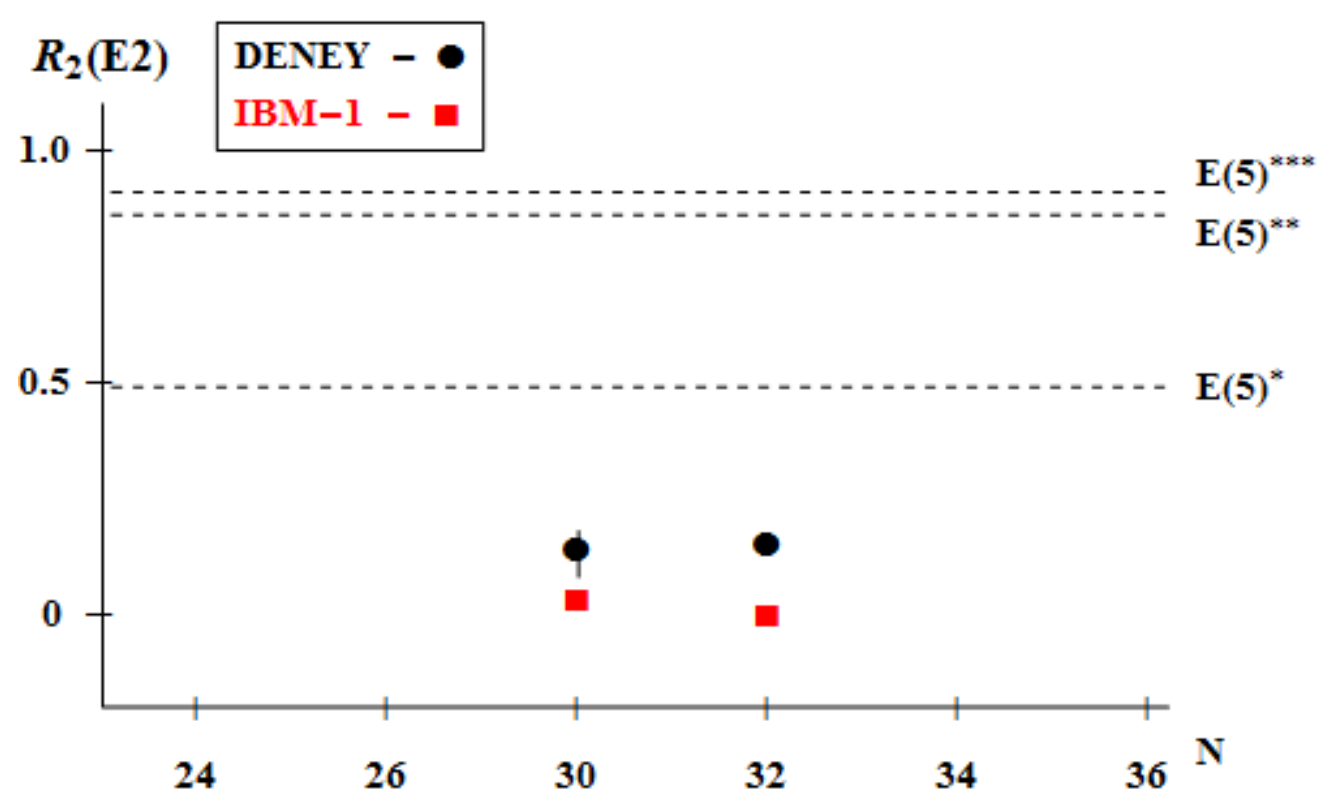

Şekil 5. ${ }^{56,58} \mathrm{Fe}$ izotoplarının $\boldsymbol{R}_{\mathbf{2}}(\boldsymbol{E} 2)=\boldsymbol{B}\left(\boldsymbol{E} 2: \mathbf{0}_{2}^{+} \rightarrow \mathbf{2}_{1}^{+}\right) / \boldsymbol{B}\left(\boldsymbol{E} 2: \mathbf{2}_{1}^{+} \rightarrow \mathbf{0}_{1}^{+}\right)$oranlarının nötron sayısına göre değişimi $(*[21], * *[18], * * *[22])$

\section{Sonuç}

Bu çalışmada, A 60 bölgesinde yer çift-çift ${ }^{50,52,56,58,60,62,64,66,68,70} \mathrm{Fe}$ izotoplarının nükleer yapı özellikleri IBM-1 modeli ile incelenmiştir. ${ }^{54} \mathrm{Fe}$ izotopunun, nötron sayısı sihirli sayı ve nötron bozon sayısı sıfir olduğu için bu çalışmaya dâhil edilmemiştir. İnceleme çerçevesinde, öncelikle model Hamiltonyeni oluşturularak, bu izotopların enerji seviyeleri hesaplanarak, deneysel veriler ile karşılaştırılarak parametrelerin uyumlu olduğu gözlemlenmiştir. Sonrasında, bu izotopların deneysel olarak B(E2) geçiş olas1l1kları hesaplanmış ve henüz deneysel verileri bilinmeyen B(E2) değerleri IBM-1 modeli ile tahmin edilmiştir. Ayrıca, bu izotopların $\mathrm{R}_{4^{+} / 2^{+}}=E\left(4_{1}^{+}\right) / E\left(2_{1}^{+}\right)$ve $\mathrm{R}_{0^{+} / 2^{+}}=E\left(0_{2}^{+}\right) / E\left(2_{1}^{+}\right)$oranları hesaplanarak, nötron sayısına göre değişimi ve $\mathrm{U}(5), \mathrm{SU}(3), \mathrm{O}(6)$ dinamik simetrilere ve de $\mathrm{X}(5), \mathrm{E}(5)$ kritik noktalarına göre şekilsel davranışı ele alınmıştır. $\mathrm{R}_{4^{+} / 2^{+}}$oranlarının değişimine göre bakıldığında, ${ }^{50} \mathrm{Fe},{ }^{56} \mathrm{Fe},{ }^{58} \mathrm{Fe},{ }^{60} \mathrm{Fe},{ }^{62} \mathrm{Fe},{ }^{64} \mathrm{Fe}$ ve ${ }^{66} \mathrm{Fe}$ izotoplarının $\mathrm{O}(6)$ limitine yakın olup $\gamma$-kararsız şekil sergilerlerken, ${ }^{52} \mathrm{Fe},{ }^{68} \mathrm{Fe}$ ve ${ }^{70} \mathrm{Fe}$ izotoplarının $\mathrm{X}(5)$ kritik noktasına yakın olup geçiş bölgesine yer aldığı görülmüştür. $\mathrm{R}_{0^{+} / 2^{+}}$enerji oranlarına göre ${ }^{56,58} \mathrm{Fe}$ izotopları $\mathrm{E}(5)$ kritik noktası civarında yer almaktadır. Enerji oranlarının yanında $\mathrm{B}(\mathrm{E} 2)$ geçiş olasılık değerlerinin $\mathrm{R}_{1}(\mathrm{E} 2)=B\left(E 2: 4_{1}^{+} \rightarrow 2_{1}^{+}\right) / B\left(E 2: 2_{1}^{+} \rightarrow\right.$ 
$\left.0_{1}^{+}\right)$ve $\mathrm{R}_{2}(\mathrm{E} 2)=B\left(E 2: 0_{2}^{+} \rightarrow 2_{1}^{+}\right) / B\left(E 2: 2_{1}^{+} \rightarrow 0_{1}^{+}\right)$oranlarının nötron sayılarına göre değişimi incelenmiştir. $R_{1}(E 2)$ oranına göre; ${ }^{52} \mathrm{Fe}$ ve ${ }^{56} \mathrm{Fe}$ izotopları $\mathrm{E}(5)$ kritik noktasına yakın yer alırken ${ }^{58,60} \mathrm{Fe}$ izotopları bu noktanın uzağındadır. Çalışmanın son kısmında ise deneysel $B\left(E 2: 0_{2}^{+} \rightarrow 2_{1}^{+}\right)$değerleri bilinen ${ }^{56} \mathrm{Fe}$ ve ${ }^{58} \mathrm{Fe}$ izotopların $R_{2}(E 2)$ oranının $\mathrm{E}(5)$ kritik noktasına göre durumu incelenmiştir. Sonuçlar genel olarak değerlendirildiğinde, 28 nötron sihirli sayısının yakınında bulunan ${ }^{56,58} \mathrm{Fe}$ izotoplarının $\mathrm{U}(5)-\mathrm{O}(6)$ geçiş bölgesinde yer aldığı görülmektedir. $\mathrm{R}_{4^{+} / 2^{+}} \mathrm{ve} \mathrm{R}_{0^{+} / 2^{+}}$enerji oranlarına göre ve $R_{1}(E 2)$ ve $R_{2}(E 2)$ geçiş oranlarına göre bu izotopların nötron sayısına göre değişimine bakıldığında, özellikle ${ }^{56} \mathrm{Fe}$ ve ${ }^{58} \mathrm{Fe}$ izotoplarının $\mathrm{E}(5)$ civarında yer alabileceği görülmektedir.

A 60 bölgesinde bulunan ve egzotik çekirdekleri de içeren bütün Fe izotopları bu çalışmada IBM-1 çerçevesinde incelenmiştir. Bu çekirdek ile aynı bölgede yer alan, $\mathrm{Cr}$ çekirdeğinin bütün çiftçift izotoplarının nükleer yapısal özellikleri QRPN ve IBM-1 ile çalışılmıştır [23,24]. Hartree-FockBogoliubov (HFB) yaklaşımı ile SLy4 ve SLy5 Skyrme kuvvetlerini hesaba dahil edilerek ${ }^{38-66} \mathrm{Ti}$ izotoplarını incelenmiş ve bu izotopların küreselden $\gamma$-kararsız şekle geçiş sergilediği ortaya konmuştur [25]. Ayrıca, çift-çift ${ }^{44-48,52-60} \mathrm{Ti}$ izotoplarının yapısal özellikleri IBM-1 modeli incelenmiş ve sonuçların deneysel veriler ile uyumlu olduğu gösterilmiştir [26]. Son yıllarda yapılan çalışmalar genel olarak değerlendirildiğinde [23-26], A 60 bölgesinde bulunan çekirdeklerin izotop zinciri boyunca kendi aralarında farklı yapısal özellikler sergilediği görülmektedir. Fe çekirdeği ve komşu çekirdeklerinin izotopları için kabuk modeli, IBM-2, QRPN, RMF gibi diğer nükleer modeller ile çalışmalar yapılmalı ve elde edilen sonuçlar kendi aralarında karşılaştırılmalıdır. Ayrıca, yapılacak olan güncel deneysel çalışmalara paralel olarak, bu ve benzer teorik hesaplamaların tekrar yapılması bu izotopların yapılarını daha iyi anlamak için önem arz etmektedir.

\section{Teşekkür}

Bu çalışma 119F127 numaralı Türkiye Bilimsel ve Teknolojik Araştırma Kurumu (TÜBİTAK) projesi ile desteklenmiştir.

\section{Yazarların Katkısı}

Çalışmaya tüm yazarlar eşit oranda katkı sunmuştur.

\section{Çıkar Çatışması Beyanı}

Yazarlar arasında herhangi bir çıkar çatışması bulunmamaktadır.

\section{Araştırma ve Yayın Etiği Beyanı}

Yapılan çalışmada araştırma ve yayın etiğine uyulmuştur.

\section{Kaynaklar}

[1] National Nuclear Data Center (NNDC).2020. https:nndc.bnl.gov (Erişim Tarihi: 15.06.2020).

[2] Bai H.B., Li X.W. 2011. Shape phase transition in neutron-rich even-even light nuclei with $\mathrm{Z}=20-28$. Chinese Physics C, 35: 925-929.

[3] Pritychenko B., Choquette J., Horoi M., Karamy B., Singh B. 2012. An update of the B(E2) evaluation for $0_{1}^{+} \rightarrow 2_{1}^{+}$transitions in even-even nuclei near $\mathrm{N} \sim \mathrm{Z} \sim 28$. Atomic Data and Nuclear Data Tables, 98: 798-811.

[4] Pritychenko B., Birch M., Singh B., Horoi M. 2016. Tables of E2 transition probabilities from the first $2^{+}$states in even-even nuclei. Atomic Data and Nuclear Data Tables, 107: 1-139.

[5] Kaneko K., Mizusaki T., Sun Y., Tazaki S. 2014. Toward a unified realistic shell-model Hamiltonian with the monopole-based universal force. Physical Review C 89, 011302 (R): 1-5.

[6] Coraggio L., Covello A., Gargano A., Itaco N. 2014. Realistic shell-model calculations for isotopic chains "north-east" of ${ }^{48} \mathrm{Ca}$ in the (N,Z) plane. Physical Review C 89, 024319: 1-12. 
[7] Kotila J., Lenzi S.M. 2014. Collective features of Cr and Fe isotopes, Physical Review C 89, 064304: 1-8.

[8] Jiao C.F., Pei J.C., Xu F.R. 2014. Beyond-mean-field calculations of collectivities of neutronrich $\mathrm{Fe}$ and $\mathrm{Cr}$ isotopes. Physical Review C 90, 054314: 1-6.

[9] Karampagia S., Zelevinsky V. 2016. Nuclear shape transitions, level density, and underlying interactions. Physical Review C 94, 014321: 1-10.

[10] Heyde K. 2004. Basic Ideas and Concepts in Nuclear Physics: An Introductory Approach. Third Edition, Institute of Physics Publishers, Bristol and Philadelphia, 1-638.

[11] Iachello F., Arima A. 1987. The Interacting Boson Model. Cambridge University Press, 1-250.

[12] Casten R.F., Warner D.D. 1988. The interacting boson approximation. Reviews of Modern Physics, 60: 389-469.

[13] Casten R.F. 2006. Shape phase transitions and critical-point phenomena in atomic nuclei. Nature Physics, 2: 811-820.

[14] Cejnar P., Jolie J. 2009. Quantum phase transitions in the interacting boson model. Progress in Particle and Nuclear Physics, 62: 210-256.

[15] Cejnar P., Jolie J., Casten R.F. 2010. Quantum phase transitions in the shapes of atomic nuclei. Reviews of Modern Physics, 82: 2155-2212.

[16] Feshbach H., Iachello F. 1973. The interacting boson model structure of ${ }^{16}$ 0. Physics Letters, 45B: 7-11.

[17] Feshbach H., Iachello F. 1974. The interacting boson model. Annals of Physics, 84: 211-231.

[18] Iachello F. 2000. Dynamic Symmetries at the Critical Point. Phys. Rev. Lett., 85: 3580-3583.

[19] Iachello F. 2001. Analytic description of critical point nuclei in a spherical-axially deformed shape phase transition. Physical Review Letters 87, 052502: 1-4.

[20] Scholten O. 1979. The program package PHINT.

[21] Arias J.M. 2001. E2 transitions and quadrupole moments in the E(5) symmetry. Phys. Rev. C 63, 034308: 1-5.

[22] Caprio M.A. 2002. Finite well solution for the E(5) Hamiltonian. Physical Review C 65, 031304(R): 1-4.

[23] Nabi J.U., Böyükata M., Ullah A., Riaz M. 2020. Nuclear structure properties of even-even chromium isotopes and the effect of deformation on calculated electron capture cross sections. Nuclear Physics A 1002, 121985: 1-19.

[24] Ullah A., Riaz M., Nabi J.U., Böyükata M., Çakmak N. 2020. Effect of deformation on gamowteller strength and electron capture cross-section for isotopes of chromium. Bitlis Eren University Journal of Science and Technology, 10: 25-29.

[25] Bayram T. 2012. An investigation on shape evolution of Ti isotopes with Hartree-FockBogoliubov theory, Modern Physics Letter A 27(28), 1250162 1-13.

[26] Şahin Y., Böyükata M. 2021. Description of even-even Ti isotopes within IBM-1 model. Cumhuriyet Science Journal, 42 (1). 